\title{
Estudo de Parâmetros do Reflexo Inibitório Retoanal em Multíparas, na Zona de Alta Pressão com Catéter Radial
}

\author{
Rectoanal Reflex Parameters With Radial Catheter \\ in The High Pressure Zone in Multípara
}

\author{
VILMAR MOURA LEAL ${ }^{1}$; CLÁUDIO SADDY RODRIGUES COY²; JOÃO JOSÉFAGUNDES²; MARIA DELOURDES \\ SETSUKOAYRIZONO $;$; JUVENALRICARDONAVARRO GÓES ${ }^{4}$
}

\author{
${ }^{1}$ Professor Assistente FACIME - UESPI, Disciplina de Coloproctologia. Cirurgião do Hospital Getúlio \\ Vargas UFPI - Aluno de Pós-Graduação FCM - UNICAMP, - SÃO PAULO - BRASIL; ; Professor Doutor - \\ Serviço de Colo-Proctologia - DMAD - UNICAMP-SÃO PAULO - BRASIL; ${ }^{3}$ Médica contratada do Serviço de Colo- \\ Proctologia - DMAD - UNICAMP-SÃO PAULO - BRASIL; ${ }^{4}$ Professor Livre-Docente - Chefe do Serviço de \\ Colo-Proctologia-DMAD - UNICAMP-SÃO PAULO-BRASIL.
}

LEAL VM; COY CSR; FAGUNDES JJ; AYRIZONO MLS; GÓES JRV. Estudo de Parâmetros do Reflexo Inibitório Retoanal em Multíparas, na Zona de Alta Pressão com Catéter Radial Rev bras Coloproct, 2006;26(3): 293-299.

RESUMO: Objetivo: analisar os parâmetros do reflexo inibitório retoanal (RIRA) em multíparas. Pacientes e métodos: 36 pacientes (40,7 anos), com quatro a seis partos normais (grupo 1) e 10 pacientes (38,5 anos) com três ou menos partos normais (grupo 2), foram submetidas à manometria com utilização do cateter radial, para pesquisar o RIRA na zona de mais alta pressão. Resultados: houve diferença significante da pressão de repouso pré-relaxamento entre os quadrantes no grupo 2 (p $=0,02)$ e da pressão no ponto de máximo relaxamento entre os quadrantes do grupo 1 (p=0,007). Comparando os grupos, não houve diferença significante entre as pressões médias de repouso $(p=\mathbf{0 , 0 5 3})$, porém esta diferença apareceu nos quadrantes posterior, lateral direito e esquerdo, quando testados separadamente, assim como entre os quadrantes posterior e lateral esquerdo no ponto de máximo relaxamento. Conclusão: diferenças radiais obtidas em alguns parâmetros do RIRA devem ser consideradas em seu estudo.

Descritores: Ânus, fisiologia, manometria, métodos.

\section{INTRODUÇÃO}

O desenvolvimento de métodos diagnósticos tem proporcionado melhor compreensão da fisiologia anorretal e dos distúrbios da evacuação, com abordagens terapêuticas mais eficazes. Dentre estes, a manometria anorretal possibilita, além das medidas de pressão de repouso e contração voluntária, a pesquisa do reflexo inibitório retoanal (RIRA). Descrito por Gowers em $1887^{16}$ e posteriormente confirmado por Denny-Brown e Robertson ${ }^{9}$ em 1935, sua importância tem sido enfatizada nos mecanismos de evacuação e continência $(18,33,44)$. Ocorrendo a distensão do reto, segue-se o relaxamento do esfíncter anal interno, permitindo contato do conteúdo retal com a porção proximal do canal anal (24). Assim, estimulam-se terminações nervosas que transmitirão a discriminação deste conteúdo, permitindo a diferenciação entre gases e fezes sólidas ou líquidas $(11,25)$.

No entanto, apesar da importância do RIRA no mecanismo da evacuação, este reflexo vem sendo pouco explorado pelos pesquisadores, sendo na maio-

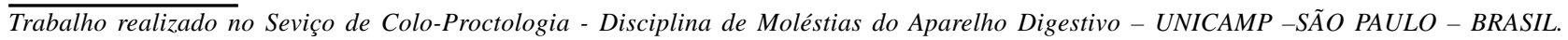

$\overline{\text { Recebido em 09/08/2006 }}$

Aceito para publicação em 29/08/2006 
ria das vezes apenas analisado como presente, ausente ou inconclusivo $(8,14,17,20,22,23,27,41,42,46)$. Recentemente, diversos autores $(15,18,19,33,34,44$, $45,47)$ vêm estudando outros aspectos do RIRA, como a amplitude de relaxamento nos diferentes níveis do canal anal, na duração e velocidade de recuperação. Nesses estudos, a pesquisa do RIRA tem sido feita com catéteres axiais, de modo que a sua observação tem sido feita em apenas um quadrante do canal anal.

Distúrbios no mecanismo de continência e de evacuação são mais freqüentes entre mulheres, principalmente após parturição, sendo a porção anterior do canal anal a região mais afetada (37). O RIRA, estudado com cateter radial de quatro canais posicionados na zona de mais alta pressão no canal anal, poderia acrescentar informações quanto ao comportamento das pressões presentes no momento do relaxamento.

\section{OBJETIVO}

Estudar o RIRA numa população de pacientes multíparas, analisando radialmente os quadrantes do canal anal quanto à intensidade do relaxamento (amplitude), à pressão no ponto de máximo relaxamento, ao tempo de relaxamento e ao tempo de recuperação.

\section{PACIENTES}

No período compreendido entre 2001 e 2002, foram estudadas 36 mulheres, com quatro a seis partos vaginais e média de idade de 40,7 anos (Grupo 1). Fazem parte do grupo controle (Grupo 2) 10 pacientes, com um a três partos vaginais e média de idade de 38,5 anos. Não houve diferença estatística na faixa etária entre os pacientes do Grupo 1 e Grupo 2 (p $=0,43)$. Com relação à função de evacuação, 63,9\% das pacientes do Grupo 1 encontravam-se assintomáticas, $33,3 \%$ apresentavam constipação como queixa e $2,8 \%$, incontinência fecal. Dentre as pacientes do Grupo 2, 50\% eram assintomáticas e as demais referiam constipação. Todas as pacientes são oriundas do Hospital Getúlio Vargas - Universidade Federal do Piauí (UFPI).

Foram excluídas pacientes submetidas a cirurgias perineais, orificiais ou a radioterapia pélvica, portadoras de doenças neurológicas, antecedentes de trauma pélvico ou perineal e com pressão anal média de repouso inferior a $30 \mathrm{mmHg}$.

\section{MÉTODOS}

As pacientes foram submetidas à manometria anorretal em decúbito lateral esquerdo, com sistema de perfusão pneumo-hidráulico (MPX816 DYNAMED), utilizando-se nitrogênio Grau Médico (G 4.6) à pressão contínua de 01 BAR em coluna de água destilada com vazão de $0,56 \mathrm{ml} /$ minuto/canal.

Ao introduzir-se o cateter no canal anal, manteve-se a correspondência do canal 1 ao quadrante posterior na linha média, o 2 ao quadrante lateral direito, o 3 ao quadrante anterior na linha média, o 4 ao quadrante lateral esquerdo. Foram então obtidos valores de pressão média de repouso (PAM) e identificouse a zona de mais alta pressão (ZAP). Os valores de pressão foram medidos em $\mathrm{mmHg}$.

A pesquisa do RIRA foi realizada na ZAP por insuflação rápida do balão de látex com ar, seguida de desinsuflação, com volume de $45 \mathrm{ml}$. A ocorrência do RIRA foi considerada positiva quando houve queda de pelo menos $25 \%$ da PAM em pelo menos um dos quadrantes.

Os parâmetros do RIRA analisados foram: pressão no ponto de máximo relaxamento (Pmax), tempo de relaxamento (Trelax) correspondente ao período compreendido em segundos entre o início do estímulo para o desencadeamento do reflexo e o ponto de máximo relaxamento da curva obtida; tempo de recuperação (Trec), período compreendido em segundos, necessário para o retorno aos valores de PAM a partir do Pmax e porcentagem de relaxamento (Prelax), diferença entre PAM e Pmax.

O presente estudo foi aprovado pelo Comitê de Ética Médica da Universidade Federal do Piauí, com assinatura pelas participantes do consentimento pósinformado.

\subsection{Análise Estatística}

Utilizou-se, para a análise dos resultados dos valores dos quadrantes do mesmo grupo, o teste não paramétrico de Friedman e para fins de comparação dos valores entre os grupos, o teste de Mann-Whitney. Consideraram-se valores estatisticamente significantes para $\mathrm{p}<0,05$.

\section{RESULTADOS}

5.1 Valores de pressão anal média de repouso pré indução do RIRA 
Não se observaram diferenças significantes da PAM entre os Grupos 1 e $2(55,03 \pm 14,62 \mathrm{mmHg}$ e $65,5 \pm 12,44 \mathrm{mmHg}$, respectivamente, $\mathrm{p}=0,053$ ) Os valores de PAM nos diferentes quadrantes estão demonstrados na tabela 1 , com significância estatística para os valores do Grupo 2. A comparação de valores nos diversos quadrantes entre os Grupos 1 e 2 foi estatisticamente diferente em todos os quadrantes com exceção do anterior (quadrante 3).

5.2 Valores médios de pressão no ponto de máximo relaxamento nos diferentes quadrantes nos Grupos 1 e 2

Os valores de Pmax foram mais baixos e com significância estatística no Grupo 1. A comparação entre os Grupos evidenciou diferença estatisticamente significante nos quadrantes 1 e 4 (tabela 2).
5.3 Porcentagem de relaxamento da pressão de repouso nos diferentes quadrantes

Não foram evidenciadas diferenças nos valores de Prelax no Grupo 1 ou Grupo 2. Na comparação por quadrantes entre os grupos, houve diferença com significância estatística no quadrante 1 (tabela 3).

\section{recuperação \\ 5.4 Tempo de relaxamento e tempo de}

Não ocorreu diferença significante com relação ao Trelax e Trec nos dois grupos, assim como a comparação das respectivas variáveis entre os grupos nos diferentes quadrantes (tabelas 4 e 5 ).

\section{DISCUSSÃO}

Os mecanismos responsáveis pela continência fecal não são ainda totalmente conhecidos, sendo

Tabela 1 - Valores de pressão anal média nos diferentes quadrantes nos Grupos 1 e 2.

\begin{tabular}{lcrl}
\hline & Grupo 1 $(\mathbf{m m H g})$ & Grupo 2 $(\mathbf{m m H g})$ & \\
\hline Quadrante 1 & $53,8 \pm 22,7$ & $76,10 \pm 11,31$ & $\mathrm{P}=0,005$ \\
Quadrante 2 & $55,86 \pm 19,42$ & $70,20 \pm 14,16$ & $\mathrm{P}=0,02$ \\
Quadrante 3 & $56,56 \pm 22,91$ & $66,10 \pm 11,97$ & $\mathrm{P}=1,65$ \\
Quadrante 4 & $52,00 \pm 22,09$ & $74,70 \pm 15,01$ & $\mathrm{P}=0,005$ \\
& $\mathrm{P}=0,37$ & $\mathrm{P}=0,02$ & \\
\hline
\end{tabular}

Tabela 2 - Pressão no ponto de máximo relaxamento.

\begin{tabular}{lccc}
\hline & Grupo 1 (mmHg) & Grupo 2 $(\mathbf{m m H g})$ & \\
\hline Quadrante 1 & $18,56 \pm 12,46$ & $28,70 \pm 8,07$ & $\mathrm{P}=0,004$ \\
Quadrante 2 & $21,50 \pm 12,51$ & $28,70 \pm 9,62$ & $\mathrm{P}=0,230$ \\
Quadrante 3 & $20,83 \pm 14,70$ & $23,20 \pm 10,21$ & $\mathrm{P}=0,438$ \\
Quadrante 4 & $17,97 \pm 14,88$ & $23,50 \pm 6,69$ & $\mathrm{P}=0,026$ \\
& $\mathrm{P}=0,07$ & $\mathrm{P}=0,26$ & \\
\hline
\end{tabular}

Tabela 3 - Porcentagem de relaxamento.

\begin{tabular}{lccc}
\hline & Grupo 1 $(\mathbf{m m H g})$ & Grupo 2 $(\mathbf{m m H g})$ & \\
\hline Quadrante 1 & $66,28 \pm 14,91$ & $62,20 \pm 9,45$ & $\mathrm{P}=0,47$ \\
Quadrante 2 & $61,61 \pm 22,29$ & $63,70 \pm 10,61$ & $\mathrm{P}=0,906$ \\
Quadrante 3 & $64,39 \pm 21,05$ & $64,90 \pm 13,28$ & $\mathrm{P}=0,927$ \\
Quadrante 4 & $65,56 \pm 20,0,6$ & $68,00 \pm 7,54$ & $\mathrm{P}=0,763$ \\
& $\mathrm{P}=0,078$ & $\mathrm{P}=0,779$ & \\
\hline
\end{tabular}


Tabela 4 - Tempo de relaxamento.

\begin{tabular}{lcrl}
\hline & Grupo 1 $(\mathbf{m m H g})$ & Grupo 2 $(\mathbf{m m H g})$ & \\
\hline Quadrante 1 & $7,11 \pm 4,62$ & $9,30 \pm 9,64$ & $\mathrm{P}=0,763$ \\
Quadrante 2 & $7,11 \pm 4,65$ & $9,30 \pm 9,64$ & $\mathrm{P}=0,763$ \\
Quadrante 3 & $6,44 \pm 4,61$ & $9,30 \pm 9,64$ & $\mathrm{P}=0,865$ \\
Quadrante 4 & $7,31 \pm 4,70$ & $9,30 \pm 9,64$ & $\mathrm{P}=0,844$ \\
& $\mathrm{P}=0,054$ & $\mathrm{P}=1,00$ & \\
\hline
\end{tabular}

Tabela 5 - Tempo de recuperação.

\begin{tabular}{|c|c|c|c|}
\hline & Grupo 1 (mmHg) & Grupo 2 (mmHg) & \\
\hline Quadrante 1 & $8,75 \pm 3,33$ & $10,50 \pm 6,82$ & $P=0,906$ \\
\hline Quadrante 2 & $8,75 \pm 3,46$ & $10,50 \pm 6,82$ & $P=0,885$ \\
\hline Quadrante 3 & $9,44 \pm 4,17$ & $10,50 \pm 6,82$ & $P=0,865$ \\
\hline Quadrante 4 & $\begin{aligned} 8,61 & \pm 3,31 \\
P & =0,109\end{aligned}$ & $\begin{array}{r}10,50 \pm \quad 6,82 \\
P=1,00\end{array}$ & $P=0,844$ \\
\hline
\end{tabular}

necessário o desenvolvimento dos métodos diagnósticos atualmente em prática ou quiçá o aparecimento de novos métodos para sua mais completa compreensão. Um dos parâmetros ainda pouco esclarecidos é o RIRA, apesar de ter sido descrito no século XIX, por Gowers ${ }^{16}$. Trata-se de componente altamente especializado, sendo que para o seu desencadeamento é necessária a integridade de várias estruturas, como o reto, o plexo mioentérico e o esfíncter anal interno. Dada a complexidade, sua real função no mecanismo da evacuação e continência fecal ainda é motivo de dúvidas.

O RIRA constitui-se basicamente por relaxamento reflexo, com componente neural intramural (20, 35). Sua melhor compreensão poderia levar, entre outras, a conseqüências práticas nos resultados funcionais das cirurgias anorretais, principalmente naquelas em que é necessária a ressecção retal. Os dados existentes na literatura com relação à pesquisa do RIRA, à percepção do neo-reto e à manutenção da continência nas cirurgias de preservação esfincteriana são controversos. $(5,12,30,31,36,39,71)$. Góes \& Beart (1995) ${ }^{15}$, em revisão da literatura sobre a fisiologia do reservatório ileal, concluíram que a presença do RIRA não seria fator determinante para a continência de pacientes submetidos à excisão total do reto. Entretanto, outros autores (29) atribuíram a distúrbios relacionados ao RIRA a ocorrência de resultados funcionais insatisfatórios no pós-operatório.
A evolução de seu estudo passou da simples constatação quanto à presença ou ausência em portadores de disfunções evacuatórias $(1,6,13,26)$, para a análise de outros parâmetros, como a intensidade do relaxamento $(15,47)$; amplitude $(4,43)$, duração (15) e velocidade de recuperação (28). Anormalidades do RIRA têm sido descritas em portadores de incontinência $(3,32,38,34,44)$ e menos freqüentemente em constipados $(2,21,28)$.

Entretanto, a pesquisa do RIRA tem sido feita sistematicamente com a utilização do cateter axial, sendo relatadas diferenças de relaxamento em vários níveis do canal anal $(15,47)$. Porém, os diferentes aspectos de seu registro têm sido obtidos em apenas um ponto do canal anal em um determinado nível. Considerando-se a assimetria da musculatura esfincteriana (40), pode-se supor que determinados aspectos do RIRA apresentar-se-iam de forma diferente nos diversos quadrantes no mesmo nível. Optouse então pelo estudo do reflexo com o cateter radial

Os dados obtidos com este cateter, nesta casuística, permitiram observações que deveriam ser levadas em conta, tendo em vista as particularidades dos Grupos estudados.

A ausência de diferença nos quadrantes do Grupo 1 dos valores de PAM, antes do desencadeamento do RIRA, pode ser atribuída ao comprometimento esfincteriano global em multíparas, com perda da assimetria presente no canal anal íntegro. A 
condição de multiparidade desta casuística não implica necessariamente em função esfincteriana comprometida, apesar da suposição de que a mesma possa ocorrer.

Considerando que valores diferentes de PAM foram obtidos no Grupo 2, poder-se-iam esperar, da mesma forma, valores diferentes nos vários quadrantes entre os dois Grupos. Entretanto, a análise comparativa desta variável foi diferente estatisticamente entre os quadrantes, exceto no anterior, possivelmente pela menor quantidade de tecido muscular presente.

A amplitude do relaxamento no RIRA, expressa neste estudo em porcentagem (Prelax), tem sido relatada como sendo mais intensa na porção proximal do canal anal $(15,47)$ quando comparada à porção distal. Zbar et al (1998) $)^{44}$ atribuíram este fenômeno a diferentes respostas da musculatura esfincteriana, em diferentes níveis do canal anal, à distensão retal. Porém não são relatados estudos comparando este fenômeno entre os quadrantes em um mesmo nível.

Diferentemente do que se poderia supor e contrariamente ao observado em relação à PAM, constataram-se, nesta casuística, diferenças significantes em relação à Pmax entre os quadrantes nas pacientes do Grupo 1, evento este não verificado no Grupo 2.

Pode-se inferir dessas observações que um canal anal mais cronicamente lesado, teria se manifestado durante o RIRA com respostas irregulares e, portanto, mais assimétricas. Em outras palavras, a normalidade do canal anal seria mais correlacionada com comportamentos mais homogêneos, quando analisados em um mesmo nível entre os quadrantes.

$\mathrm{Na}$ comparação da Pmax entre os quadrantes dos dois Grupos, constatou-se diferença significativa nos quadrantes 1 e 4 (menores no Grupo 1). A interpretação das causas para estes achados não foi possível, analisando-se apenas os dados manométricos.
A constatação nesta casuística de que certos dados obtidos, apesar de se apresentarem com diferenças estatisticamente significativas, a correlação com a clínica é difícil e talvez não estejam necessariamente em acordo com alterações morfológicas. Pode-se supor que a conhecida assimetria anatômica do canal anal não corresponderia, do ponto de vista prático, às diferenças obtidas em alguns parâmetros do RIRA estudados nesta casuística

A importância da musculatura lisa anal nos mecanismos de continência fecal tem sido cada vez mais considerada, principalmente nas situações em que há comprometimento da musculatura estriada. O estudo de parâmetros do RIRA e a sua melhor compreensão poderia contribuir de forma prática, possivelmente predizendo resultados funcionais em pacientes que serão submetidos a cirurgias orificiais ou de ressecção retal, com função esfincteriana limítrofe. Assim, com a utilização do cateter radial, sofisticar-se-ia o estudo do RIRA, permitindo a obtenção de informações adicionais não disponíveis quando a sua pesquisa é feita com cateter axial.

\section{CONCLUSÕES}

Os resultados obtidos nesta casuística permitem concluir que:

1 - A PAM tende a uma queda da assimetria nas pacientes multíparas, provavelmente decorrente de comprometimento global da função esfincteriana.

2 - O RIRA, contrariamente à PAM, pode ter os parâmetros que o compõem manifestados de maneira irregular nos diferentes quadrantes da ZAP, da mesma forma, em decorrência das alterações estruturais mais possíveis nas pacientes do Grupo 1

3 - A utilização do cateter radial no estudo do RIRA pode fornecer dados não obtidos com cateter axial.

\footnotetext{
ABSTRACT: Purpose: Rectoanal reflex parameters were analyzed in multiparous. Methods: Thirty-six patients with 4 to 6 (Group 1) and 10 patients with 1 to 3 (Group2) vaginal deliveries were submitted to anal manometry with four channel radial catheter. Various parameters of the rectoanal inhibitory reflex were analyzed in each quadrant at the anal canal highest pressure zone. Results: There were significant differences in the anal pressures at the point of maximum relaxation curve among quadrants in Group 1 as well as with the anal resting pressure in Group 2. The comparision between Groups showed differences in anal resting pressure in posterior right lateral and left lateral quadrants as well as in posterior and left lateral at the maximum relaxation point. Conclusion: Radial differences in the anal pressure before relaxation and in rectoanal inhibitory reflex parameters are present; however there is not an obvious relevance under the clinical point of view.
} 


\section{REFERÊNCIAS}

1. Aspiroz F, Enk P, Whitehead WE. Anorectal function testing: review of collective experience. Am J Gastrenterol 2002; 97(2):232-40.

2. Baldi DC, Ferrarini F, Corinaldesi R. Function of the internal anal sphincter and recta sensivity in idiopathic constipation. Digestion 1982; 24: 14-22.

3. Banister J, Read NW, Donelly TC, Sun WM. External and internal anal sphincter responses to rectal distension in normal subjects and in patients with idiopathic faecal incontinence. Br J Surg 1989; 76: 617-21.

4. Bartolo DCC, Bartram CI, Ekberg O, Fork FT, Kodner L, Kuijpers JH, et al. Symposium Proctograpy. Int J Colrectal Dis 1988; 3(2):67-89.

5. Beart RW Jr, Dozois RR, Wolff BG, Pembertom JH. Mechanisms of rectal continence: lessons from the ileoanal procedure. Am J Surg 1985; 149:31-4.

6. Coller. Application of anorectal manometry. Gastronterol Clin North Am 1987; 16(1):17-33.

7. Coy CSR, Goes JRN, Fagundes JJ, Ayrizono MLS, Shoji N, Medeiros RR. Análise de resultados funcionais e parâmetros fisiológicos nas anastomoses coloanais com e sem reservatório. Rev Bras Coloproct 1999; 19:83-8.

8. Davies MR, Cywes S, Rode H. The manometric evaluation of the rectosphincteric reflex in total colonic aganglionosis. J Pediatr Surg 1981; 16: 660-3.

9. denny-brown D, Robertson EG. An investigation of the nervous control of defecation. Brain 1935; 58:256-310.

10. DUTHIE HL, WATTS J.M. Contribution of the external anal sphincter to the pressure zone in the anal canal. Gut 1965; 6:64-8.

11. Duthie HI, Bennett RC. The relation of sesation in the anal canal to the functional anal sphincter: a possible factor in anal continence. Gut 1963; 4:179-82.

12. Emblem R, Stien R, Morkrid L. Ananl sphincter function after colectomy, mucosal protectomy, and ileoanalanastomosis. Scand J Gastroenterol 1989; 24(2):171-8.

13. Faiguel DO. A clinical approach to constipation. Cli Cornerstone 2002; 4(4):1-11.

14. Frenckener B, Von Euler C. Influence of pudendal block on the anal sphincters. Gut 1975; 16:482-9.

15. Goes JRN, Simons AJ, Masi L, Beart RW JR. Gradient of pressure and time between proximal anal canal and highpressure zone during internal anal sphincter relaxation: its role in the fecal continence mechanism. Dis Colon Rectum 1995; 38:1043-46.

14. Gowers WR. The automatic action of the sphincter ani. Proc. R. Soc. Lond 1877; 26:77-84.

15. Habr-Gama A, Raia A, Correa Neto A. Motility of the sigmoidcolon and rectum, contribution to the physiopahology of megacolon in Chagas disease. Dis Colon rectum 1971; 14:291-304.
16. Ihre T. Studies on anal function in continent and incontinent patients. Scand J Gastroenterol 1974; 9 suppl 25: 1-64.

17. Kaur G, Gardiner A, Duthie GS. Rectoanal reflex parameters in incontinence and constipation. Dis Colon Rectum 2002; 45:928-33.

18. Lawson JO, Nixon HH. Anal canal pressures in the diagnosis of Hirschsprung's disease. J Pediatr Surg 1967; 2:544-52.

19. Loening-Baucke VA, Younouszai MK. Abnormal and sphincter response in chronically constipated children. J Pediatr 1982;100: 213-8.

20. Lubowski DZ, Nicholls RJ, Swash M, Jordan MJ. Neural control of internal anal sphincter function. Br J Surg 1987; 74:668-70.

21. Lubowski DZ, Swash M. Effect of sympatathetic innervation on the human internal anal sphincter. Int J Colorectal Dis $1991 ; 6: 175-6$.

22. Mavrantonis C, Wexner SD. A clinical approach to fecal incontinence. Clin Gastroenterol 1998; 27(2):108-21.

23. Miller R, Bartolo DCC, Cervero F, Mortensen N.J. Anorectal sampling: a comparision of normal and incontinent patients. Br J Surg 1988; 75:44-7.

24. Minguez M, Moreno-Osset E, Tomas-Ridocci M, Mora F, Benages A. Alteration of rectoanal motility in chronic idiopathic constipation. Ital J Gastroenterol 1991; 23(8) suppl 1:16-9.

24. Nagasaki A, Ikeda K, Suita S, Sumitomo K. Induction of the rectoanal reflex by electric stimulation: a diagnostic aid for Hirschsprung's disease. Dis Colon Rectum 1984; 27:598-601.

25. Netinho JG. Intensidade e duração da recuperação do relaxamento esfincteriano induzido pelo reflexo inibitório retoanal e sua correlação com constipação por evacuação obstruída. [Tese de doutorado]. São Paulo: UNICAMP. 2002

26. Otto IC, Ito K, Ye C, Hii K, Kasai I, Akiyama S, et al. Causes of rectal incontinence after sphincter-preserving operations for rectal cancer. Dis Colon Rectum 1996; 39:1423-7.

27. PescatorI M, Parks AG. The sphincteric and sensory components of preserved continence after ileoanal reservoir. Surg Gyecol Obstet 1094; 158(6):517-21.

28. Sagart PM, Holdsworth PJ, Johnston D. Correlation between laboratory findings and clinical outcome after restorative proctocolectomy: serial studies in 20 patients with end-toend pouch-anal anastomosis. Br J Surg 1991; 78:67-70.

29. Sangwan YP, Coller JA, Schoetz DJ JR, Murray JJ, Roberts PL. Latency measurement of rectal anal reflexes. Dis Colon Rectum 1995; 38:1281-5.

30. Sangwan YP, Coller JA, Barrett RC, Murray JJ, Roberts PL, Schoetz DJ JR. Distal rectoanal excitatory reflez: a reliableindex of pudendal neuropathy? Dis Colon Rectum 1995; 38:916-20.

31. Sangwan YP, Coller JA, Schoetz DJ JR, Roberts PL, Murray JJ. Spectrum of abnormal rectoanal reflex patterns in patients with fecal incontinence. Dis Colon Rectum 1996; 39:59-65.

32. Schnaufer L, Talbert JL, Haller JA, Reid NC, Tobon F, Schuster MM. Differential sphincter studies in the diagnosis of anorectal disorders of childhood. J Pediatr Surg 1967; 2:538-43. 
33. Stryker SJ, Kelly KA, Phillips SF, Dozois RR, Beart RWJr. Anal and neorectal function after ileal pouch-anal anastomosis. Ann Surg 1986; 203:55-61.

34. Sultan AH, Kamm MA, Hudson CN. Anal-sphincter disruption during vaginal delivery. N Engl J Med 1993; 329:1905-11.

35. Sun WM, Read NW, Miner PB. Relation between rectal sensation and anal function in normal subjects and patients with fecal incontinence. Gut 1990; 31:1056-61.

36. Suzuki H, Matsumoto K, Amano S, Fujioka M, Honzumi M. Anorectal pressure and rectal compliance after low anterior ressection. Br J Surg 1980; 655-7.

37. Taylor BM, Beart RW, Phillips SF. Longitudinal and radial variations in pressure in the human anal sphincter. Gastroenteroly 1984; 86:693-7.

38. Tobon F, Reid NC, Talbert JL, Schuster MM. A manometric test for the diagnosis of Hirschsprung's disease. Gastroenterol 1967; 52:1143.

39. Tobon F, Reid NC, Talbert JL, Schuster MM. Nonsurgical test foe the diagnosis of Hirschsprung's disease. N Engl J Med 1968; 278:188-94.

40. Touchais JY, Ducrotte P, Weber J, Louvel JP, Martin PA, Benozio $\mathrm{M}$, et al. Relationship between results of radiological pelvic floor study and anorectal manometry in patients colsulting for constipation. Int J Colorectal Dis 1988; 3(1):53-8.
41. Zbar AP, Aslam M, GOLD DM, Garzen C, Goslinga, Kmiot WA. Parameters of the rectoanal inhibitory reflex in patients with idiopathic fecal incontinence and chronic constipation. Dis Colon Rectum 1998; 41(2):200-8.

42. Zbar AP, Beer-Gabel M, Chiappa AC, Aslam M. Fecal incontinence after minor anorectal surgery. Dis Colon Rectum 2001; 44:1610-9.

43. Wald A, Tunuglunta AK. Anorectal sensorimotor dysfunction in fecal incontinence and diabetes mellitus: Modification with biofeedback therapy. N Engl J Med 1984; 310:1282-7.

44. Williamson JL, Nelson RL, Orsay C, Pearl RK, Abcarian H. A comparison of simultaneous longitudinal and radial recordings of anal canal pressures. Dis Colon Rectum 1990; 33:201-6.

\section{Endereço para correspondência:}

\section{VILMAR MOURA LEAL}

Rua Tabelião José Basílio, 1248 - Bairro de Fátima

64.048-190 - Teresina (PI)

Telefone: (0xx86) 3233-4789 / Fax: (0xx86) 3223-7315

E-mail: vilmarmoura@yahoo.com.br

vilmarmoura@hotmail.com

vilmarml@ufpi.br 